\title{
Ein Rettichöl-Darlehen in Hamburg und Berlin
}

\author{
Mit Abb. S. 144
}

Dieter Hagedorn (Heidelberg) und Günter Poethke (Berlin)

Durch einen bemerkenswerten Zufall hat sich ergeben, daß Bruchstücke ein und desselben Vertrags, die seit mehreren Jahrzehnten in den Papyrussammlungen der Staats- und Universitätsbibliothek Hamburg und der Staatlichen Museen zu Berlin getrennt aufbewahrt wurden, im Abstand von nur drei Jahren nacheinander erstmals ediert worden sind. Es handelt sich um die beiden Fragmente P.Hamb. IV $266^{1}$ einerseits und BGU XVII $2688^{2}$ andererseits. Letzteres (P.Berol. 11836) ${ }^{3}$ schlieBt unmittelbar an das Fragment A von P.Hamb. IV 266 an. Der Bruch verläuft $z$ wischen $Z$. 7 und $Z$. 8 der Urkunde dergestalt, daß - wie die Photomontage $S$. 144 erkennen läßt - mehrere Unterlängen der Buchstaben von Z. 7 bzw. Oberlängen der Buchstaben von Z. 8 sich jenseits der Bruchkante fortsetzen. Zwischen BGU XVII 2688 und Fr. B von P.Hamb. IV 266 hingegen fehlen anscheinend immer noch mehrere Zeilen. Dies erweckt die Hoffnung, daß bisher nicht entdeckte weitere Fragmente noch hinzugefunden werden, sei es in Hamburg, Berlin oder an einem dritten Ort.

Wir geben hier eine Neuedition der virtuell vereinigten Fragmente, wobei wir uns im Kommentar auf wenige Bemerkungen beschränken, die durch die Zusammensetzung notwendig geworden sind, und im übrigen auf die Ersteditionen verweisen.

${ }^{1}$ B. Kramer, D. Hagedorn, Griechische Papyri der Staats- und Universitätsbibliothek Hamburg (P.Hamb. IV). (APF Beiheft 4), Stuttgart - Leipzig 1998, Tafel XXVI.

${ }^{2} \mathrm{G}$. Poethke, Griechische Papyrusurkunden spätrömischer und byzantinischer Zeit aus Hermupolis Magna (BGU XVII). (APF Beiheft 7), München - Leipzig 2001, Tafel XVI.

3 Hierzu findet sich im Berliner Inventarbuch folgende Bemerkung: "Angekauft durch Dr. F. Zucker 1910". Friedrich Zucker grub zusammen mit Wilhelm Schubart im Winter 1909/10 in Dîme. Möglicherweise hat er das Fragment bei dieser Gelegenheit erworben. Im Erwerbungsjournal der Hamburger Sammlung steht nach Auskunft von E. Horvath bei Inv. Nr. 600 nur die Akz.-Nr. 35. 2774.5 und folgende Notiz: "griech. Pap. aus den alten Papyruskäufen, von Herrn Ibscher verglast". 

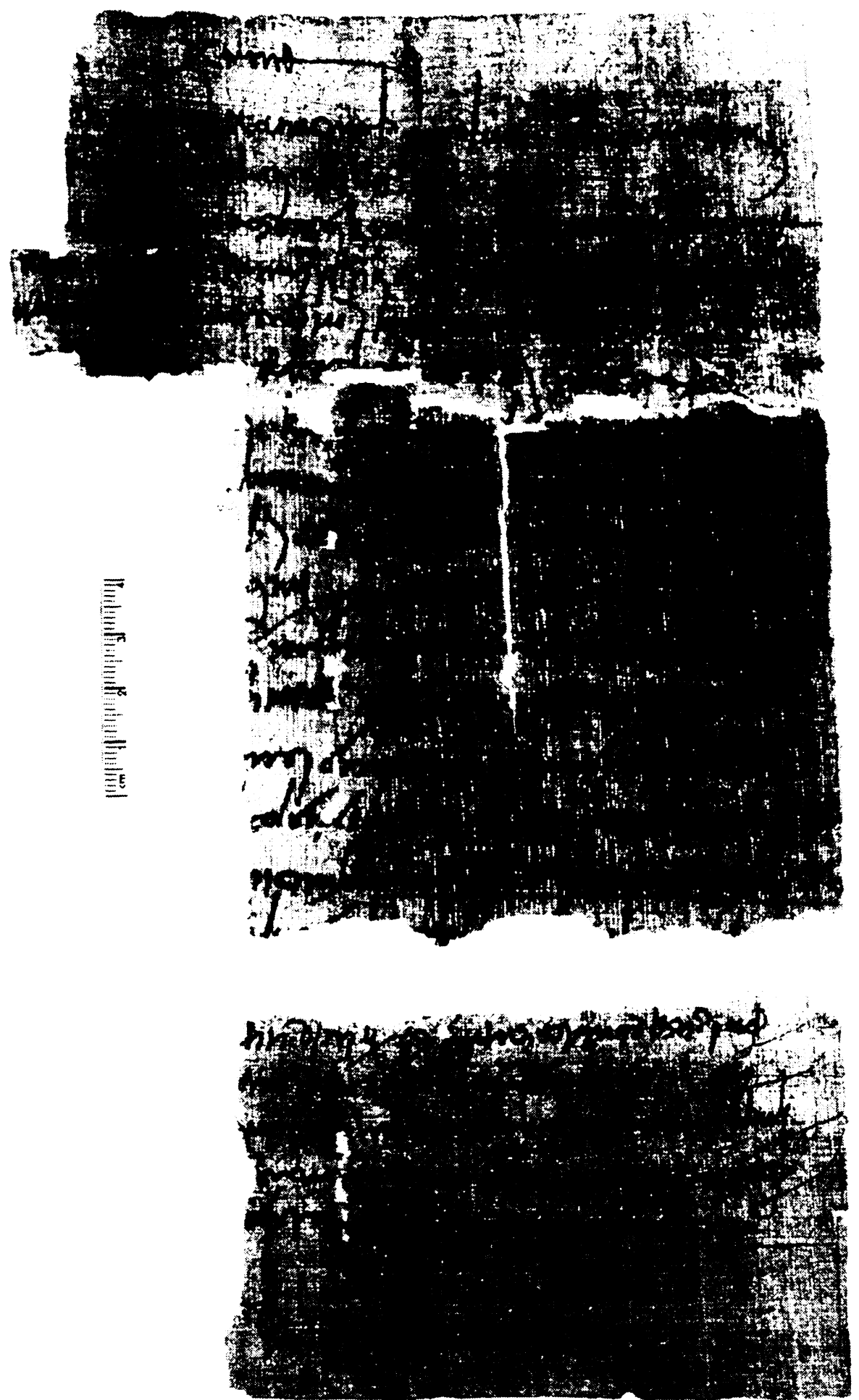


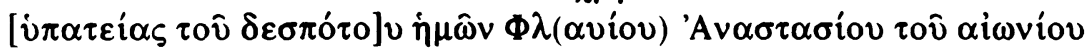

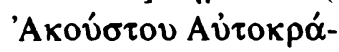

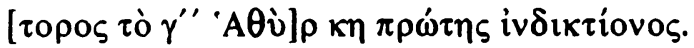

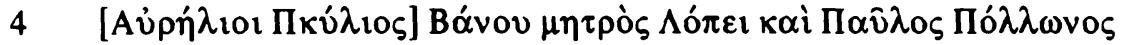
$\mu \eta \tau \rho o ̀ s ~ M \alpha \rho i ́ \alpha$

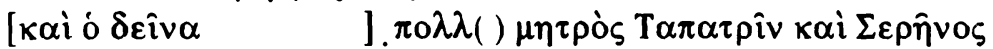

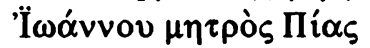

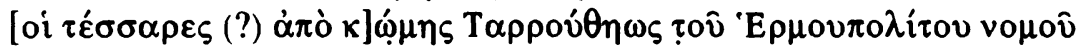

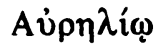

[

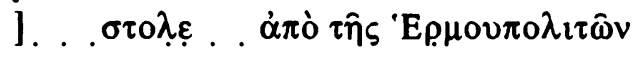
$\chi(\alpha i) \rho(\varepsilon \mathbf{i v})$.

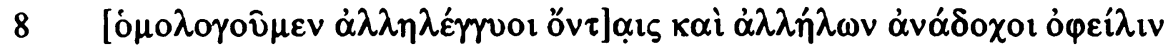
oot

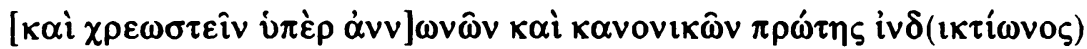

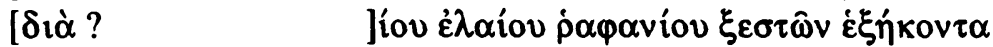

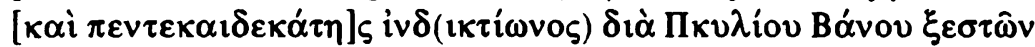

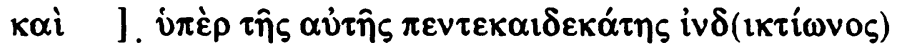

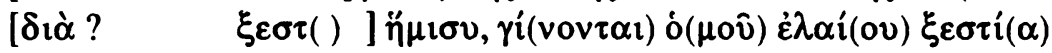
$\pi \gamma /$, پँ $\pi \varepsilon \rho$

[ ] ] ] ]

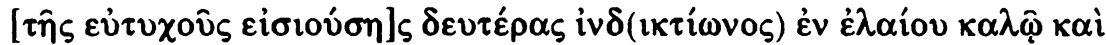
$\varepsilon \dot{\alpha} \alpha \varepsilon \dot{\sigma} \sigma \varphi$

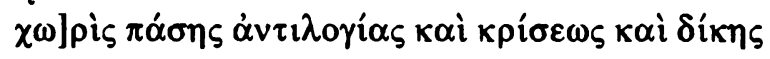

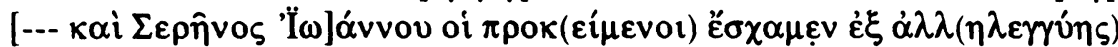

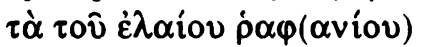

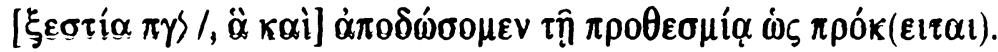

$A \dot{v} \rho\left(\eta^{\lambda} \lambda_{101}\right)$

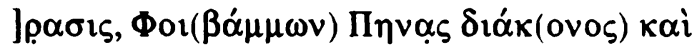

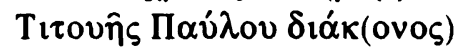

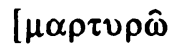

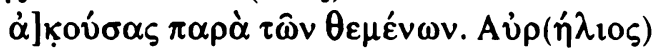

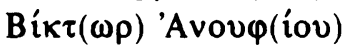

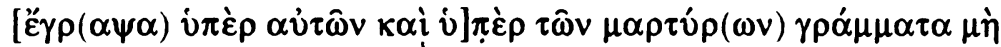
$\varepsilon i \delta o ́ \tau(\omega v)$.

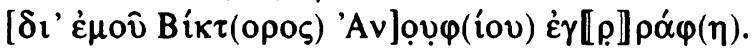

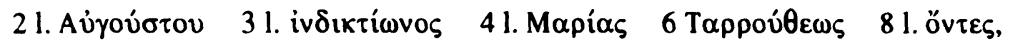

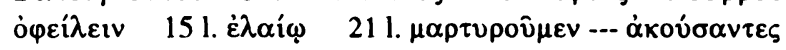




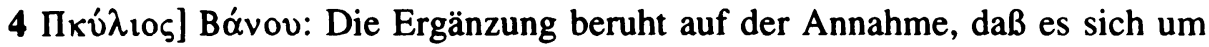
dieselbe Person handelt wie die in Z. 11 genannte. Falls dies nicht zutrifft, käme weiterhin für den Vatersnamen auch die Ergänzung $\Sigma \imath \lambda] \beta \alpha v o v$ in Betracht.

10 ]íov ist wohl das Ende des Vatersnamens des ersten Schuldners; vgl. Z. 11

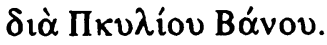

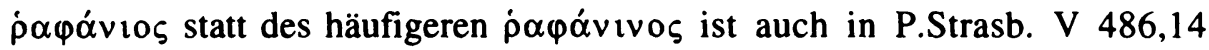
(Herm.; 549/50 n.Chr.) überliefert; in Z. 18 lösen wir die Abkürzung $\rho \alpha \varphi()$ daher ebenso auf.

$13 \mathrm{Zu}$ der Abkürzung - bisweilen auch $\emptyset$ geschrieben - für ó $(\mu \circ \hat{v})$ vgl. z.B. P.Lond. V, S. 322 mit Verweis auf P.Lond V 1654,1 und 1668,10.

Das Deminutiv $\xi_{\varepsilon \sigma \tau i ́ o v}$ findet sich auch in CPR VIII 70,10; P.Oxy. XVI 1862,11b; SB VIII 9778,12; SB XII 10808,4; SPP VIII 1100,4 u.a. Vgl. auch hier in Z. 18 den Artikel des Neutrums $\tau$ q́.

\section{Übersetzung}

Gott ist Helfer (?), Amen.

Im Konsulat unseres Herren Flavius Anastasius perpetuus Augustus Imperator zum dritten Mal, am 28. Hathyr der ersten Indiktion.

Die Aurelier Pkylios, Sohn des Banos und der Lopei, und Paulos, Sohn des Pollon und der Maria, und -.-, Sohn des -poll() und der Tapatrin, und Serenos, Sohn des Johannes und der Pia, die vier (?) aus dem Dorf Taruthis des Gaues von Hermupolis, grüßen Aurelios --- aus Hermupolis.

(Wir erklären, indem wir für uns wechselseitig bürgen) und wechselseitig haften, dir für die Annona und Kanonika der ersten Indiktion zu schulden durch -.sechzig Sextarii Rettichöl und für die fünfzehnte Indiktion durch Pkylios, den Sohn des Banos, --- Sextarii und für dieselbe fünfzehnte Indiktion durch --- und einen halben Sextarius, ergibt zusammen 83 1/2 Sextarii Öl, die wir bereit sind, dir im Monat Epeiph der mit Glück kommenden zweiten Indiktion in gutem und einwandfreiem Öl zu liefern ohne jede Widerrede und Urteil und Rechtsspruch ---

--- und Serenos, der Sohn des Johannes, die vorgenannten, wir haben für einander bürgend die [ $83 \quad 1 / 2$ Sextarii] Rettichöl erhalten, die wir termingerecht zurückgeben werden, wie voransteht.

--- Wir, die Aurelier ---, Phoibammon, Sohn des Penas (?), Diakon, und Tituës, Sohn des Paulos, Diakon, bezeugen die Vereinbarung, wie wir von den Vertragspartnern gehört haben.

Ich, Aurelios Biktor, Sohn des Anuphis, habe für sie und die Zeugen geschrieben, da sie nicht schreiben können. $†$

Geschrieben durch mich, Biktor, den Sohn des Anuphis. 